Western University

Scholarship@Western

Fall 9-24-2017

\title{
Grasp Evaluation Method for Applying Static Loads leading to Beam Failure
}

Mahyar Abdeetedal

Amazon

Mehrdad Kermani Ph.D., P.Eng.

Western University, mkermani@eng.uwo.ca

Follow this and additional works at: https://ir.lib.uwo.ca/electricalpub

Part of the Computer Engineering Commons, and the Electrical and Computer Engineering Commons

Citation of this paper:

Abdeetedal, Mahyar and Kermani, Mehrdad Ph.D., P.Eng., "Grasp Evaluation Method for Applying Static Loads leading to Beam Failure" (2017). Electrical and Computer Engineering Publications. 547.

https://ir.lib.uwo.ca/electricalpub/547 


\title{
Grasp Evaluation Method for Applying Static Loads leading to Beam Failure
}

\author{
Mahyar Abdeetedal and Mehrdad R. Kermani ${ }^{1}$
}

\begin{abstract}
This paper deals with the problem of purposefully failing or yielding an object by a robotic gripper. We propose a grasp quality measure fabricated for robotic harvesting in which picking a crop from its stem is desired. The proposed metric characterizes a suitable grasp configuration for systematically controlling the failure behavior of an object to break it at the desired location while avoiding damage on other areas. Our approach is based on failure task information and gripper wrench insertion capability. Failure task definition is accomplished using failure theories. Gripper wrench insertion capability is formulated by modeling the friction between the object and gripper. A new method inspired by human premanipulation process is introduced to utilize gripper itself as a friction measurement device. The provided friction model is capable of handling the anisotropic behavior of materials which is the case for fruits and vegetables. The evaluation method is formulated as a quasistatic grasp problem. Additionally, the general case of both fully-actuated and under-actuated grippers are considered. As a validation of the proposed evaluation method, experimental results for failing parts using Kuka LightWeight Robot IV robot are presented.
\end{abstract}

\section{INTRODUCTION}

Harvesting is the process of gathering a ripe crop through failing it into pieces at desired location. This process has to be systematically controlled to avoid any damage to the target fruit or vegetable. The complete separation of an anisotropic beam such as a fruit stem or a tree branch is difficult to model. Buckling and green-stick fracture in biological beams can avoid the beam from snapping. This behavior can be explained by anisotropy between the radial and tangential directions of fiber cells [1]. We propose a grasp evaluation method dedicated to the systematic failure of an object which takes mechanical and physical properties of the material into account.

Over the last four decades, significant contributions have been made in the field of robotic grasping [2], [3], [4]. As massively reported in the literature, a grasp task can vary from the simple pick and place robots, to more sophisticated assembly such as circuit chips insertion. In robotic harvesting, failure of the grasped object at a certain location is desired. To the best of our knowledge, there is no investigation on grasp planning of a grasped object failure. Even the works which consider avoiding deflection and/or slippage of the object [5], [6], [7] do not study the individual effects of bending, tension, or torsion on the object which is essential for obtaining an accurate characterization of object failure grasp task.

\footnotetext{
${ }^{1}$ Mahyar Abdeetedal and Mehrdad R. Kermani are with Faculty of Electrical and Computer Engineering, Western University , London, Ontario, Canada mabdeete@uwo.ca, mkerman2@uwo.ca
}

Grasp tasks can be characterized by a set of expected wrenches that the grasp must withstand while being manipulated [8]. A task polytope can be defined using all these wrenches [9] which are also called Task Wrench Space (TWS). A TWS can be approximated by an ellipsoid [10] or a convex polytope [11]. A grasp can be evaluated using TWS, for instance, a well-known task-oriented grasp metric is to choose an appropriate TWS and then measure how well it can be inscribed in a grasp wrench tolerance [12]. The core of our approach involves computing the maximum force that can be applied to a grasped object which draws upon optimizing contact forces and analyzing force capabilities. Our primary focus is on planning a grasp for yielding a tensile object or fracture of a brittle object.

In this paper, we suggest defining a failure grasp task using mechanical failure theories; then evaluate the grasp that how well the TWS matches with the capabilities of the gripper. The grasp capability is formulated using wrench insertion capability of the gripper and the friction between the part and the gripper. Friction can play a major role, since bending moment, tension force and torsion torque require contact points with friction when form closure is not achievable. It is common in the literature that Coulomb's law to be used to model the dynamic friction force between the gripper and object [2]. However, friction in anisotropic materials such as fruits can vary significantly and cannot be characterized using a single Coulomb's friction coefficient. Studies have demonstrated that humans adapt their exploratory movements in ways that may improve information gained through mechanical stimuli elicited during such interactions [13], [14], [15]. We are inspired by this natural approach to use gripper itself as a friction measurement device during object manipulation. Our contributions in this paper are as follows:

- A novel failure task definition is introduced to be used in grasp evaluation method. Mechanical failure theories are carefully selected for any brittle or ductile materials for accurate failure behavior prediction.

- A new friction identification process is designed to be fast and easy to implement. It suggests moving the gripper on the object in few different directions to obtain an anisotropic friction behavior of the object using the proposed model. We capture frictional data using the gripper to be then able to formulate the gripper wrench insertion capability.

- Task oriented grasp evaluation method considering the gripper capability is proposed. Since the gripper capability is dependent on its actuation system, both cases 
of the fully-actuated and under-actuated gripper are considered.

The structure of this paper is as follows: In Section II presents the failure grasp task definition. Section III introduces a grasp evaluation method. Section IV investigates the validity of the presented approach via experimental results.

\section{FAILURE GRASP TASK DEFINITION}

In this paper, failure is interpreted as a brittle part to be separated into two or more pieces and a ductile part to become permanently distorted. Failure theories help mechanical designers to immune their designs from failure. These theories provide the minimum principle stresses which are just enough to fail the part. Failure theories are conservative over not reaching the object stress tolerance. In this paper, failure theories are used to ensure the lowest effort for purposefully failing a beam. There are several theories for each type of material (ductile or brittle) formulating the failure behavior. In this paper, a careful selection of these theories and failure task definition are made based on the following assumptions

- A material that normally is considered as ductile fails in a ductile manner.

- All materials are considered to have equal ultimate strengths in tension and compression.

- A beam with any general profile requires less stress to fail than a virtual cylinder covering it.

The first assumption is valid when there are no cracks in the object, and manipulation temperature is higher than the transition temperature which prevents sudden brittle fracture of so-called ductile material. Note that yielding a ductile object can cause cracks in it [16]. Our interest in considering the ductile materials here is based on the fact that if biological beams acts in a ductile manner, then they can be snapped in a brittle manner after yielding. The second assumption is used for the sake of simplicity even though there are rare cases in which ultimate strengths in tension and compression are unequal (e.g. magnesium alloys). The third assumption is the generalization of the target object profile. This assumption guarantees the object failure since failure theories are conservative about not failing the object while the virtual beam requires larger stress to fail due to its larger diameter for covering the original beam.

For ductile behavior, the selected criterion is the distortionenergy theory. Maximum shear stress theory and ductile Coulomb-Mohr theory are not applied since they are too conservative and suitable for unequal yield strengths, respectively [17]. The distortion-energy theory predicts that yielding occurs when the distortion strain energy per unit volume reaches or exceeds the distortion strain energy per unit volume for yield in simple tension or compression of the same material. For brittle behavior, we choose modified Mohr over brittle Coulomb-Mohr since it is less conservative. The modified Mohr theory states that failure occurs whenever one of the principal stresses equals or exceeds the ultimate strength.
In order to be able to apply normal and shear stresses by a normal-sized conventional robot, leverage based stresses are applied to deliver the highest impact of the robot to the part. The normal stress for a circular beam subjected to a bending moment, $M_{b}$, can be obtained by $\sigma_{n}=\frac{M_{b} c}{I}$ where $I$ is the second moment of area, and $c$ is the radius to outer beam surface. The shear stress resulted by twisting moment, $M_{t}$, acting on the same beam is given by $\sigma_{t}=\frac{M_{t} c}{P}$ where $P$ is the polar second moment of area. Having normal and shear stresses, task definition for the purpose of failing the object is possible.

Based on Krein-Milman theorem, vertices of convex hull that bounds the TWS can be used for grasp task definition. In object failure, task wrenches are generated by the object reaction during stress application. Failing/yielding wrench vector $w_{y}=\left[0,0,0, M_{b}, 0, M_{t}\right]$ is enough for failing the object if it results in normal and shear stresses satisfying the distortionenergy theory and modified Mohr theory for ductile, and brittle materials, respectively. Mathematically,

Proposition 1: Wrench vector $w_{y}$ fails/yeilds a ductile object if $\sigma^{\prime}>S_{y}$ where $\sigma^{\prime}=\sqrt{\sigma_{A}^{2}-\sigma_{A} \sigma_{B}+\sigma_{B}^{2}}$ is von Mises stress, $\sigma_{A}$, and $\sigma_{B}$ are principal stresses.

Proposition 2: Wrench vector $w_{y}$ fails/yeilds a brittle object if $\sigma_{A} \geq S_{u}$ or $\sigma_{B} \leq-S_{u}$ where $S_{u}$ is ultimate strength of the object.

Note that we can always change the grasp configuration and map any wrench to the point of interest, therefore, the planar principle stresses are considered without loss of generality.

Consider the grasped object in Fig. 1 which we want to fail it at the point of interest $O$. Manipulation during quasistatic motions can be expressed as,

$$
w=-G f
$$

where $w \in R^{6}$ is the wrench exerted on the object by gravity and/or external sources, $G \in R^{6 \times 3 n_{c}}$ is the Grasp matrix, $f \in R^{3 n_{c}}$ is contact forces vector, and $G f$ is the total wrench applied to the object by the gripper. The Grasp matrix maps the transmitted contact forces and moments to the set of wrenches that the gripper can apply to the object origin using moment arm vector, $r_{o i}$, for each contact (see Fig. 11. For failing the object in Fig. 1, contact forces which are mapped to the origin and result in wrench balance with object reaction must be found. The general solution of (1) is $f=-G^{+} w+\mathrm{A} \xi$ where $G^{+}$is assumed to be right inverse of Grasp matrix, and $\mathrm{A} \in R^{3 n_{c} \times g}$ is a matrix whose column spans the subspace of Grasp matrix nullspace $(\mathscr{N}(G))$ excluding the left nullspace of the gripper Jacobian matrix, and $\xi \in R^{g}$ is a free $g$-vector which parametrizes the homogeneous solution. The contact forces are constantly being adjusted according to the external wrenches. In quasistatic condition, same wrench vector as failure wrench is reacted by the object to keep the balance (1) until the object fails.

According to our experiments, bending produces a larger portion of failing/yielding stress with less deflection since it is primarily leveraged with the length of the beam [18]. 


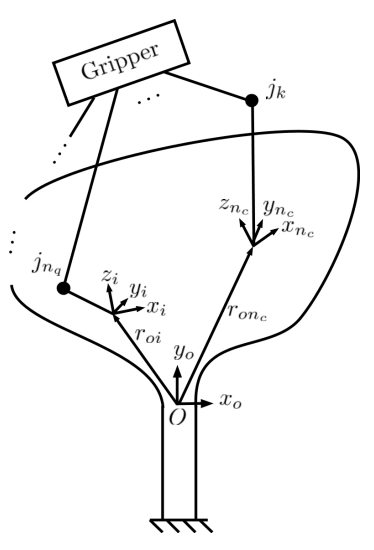

Fig. 1. Mapping from the transmitted $n_{c}$ contact points forces and moments $\left(r_{o i}\right)$ to the set wrenches that the gripper can apply to the object at $O$, and a mapping from the transmitted contact forces and moments to $j_{k}$ load of the $n_{q}$ joints.

One notable exception is harvesting, in which the volume of the fruit provides a long arm for applying torsion to the stem which results in relatively large shear stress (see Fig. 1). Imagine that the object in Fig. 11 is a fruit and we are interested in breaking it at point $O$. Large moment arm vector, $r_{o i}$, provides higher leverage for twisting as well as bending. Therefore, the shear stress resulted by twisting will be comparable to bending normal stress. Note that there are cases of fruit clusters that there is not enough room for bending, hence twisting will be a suitable alternative.

\section{Grasp Planning Method}

A fully defined failure task enables planning the grasp. The interest location for fracture in such tasks results in a small number of candidate grasps. The capability of the gripper also has to be taken into account for the selection among the remaining grasp candidates. The actuation saturation limit and the contacts friction restrict the capability of the gripper.

\section{A. Friction Identification}

Contact points with friction are necessary for applying tangential force and avoiding slippage. Coulomb's friction law states the relation between the tangential component of contact force, $f_{t i}$, and its normal component, $f_{n i}$, i.e. $\left\|f_{t i}\right\|=\mu\left\|f_{n i}\right\|$ where $\mu$ is the friction coefficient. However, this model is not suitable for robotic harvesting where crops have high anisotropic behavior. Therefore, We propose the following friction model based on experimental friction data

$$
\left\|f_{t i}\right\|=u^{T}\left[\begin{array}{ll}
\mu_{x x} & \mu_{x y} \\
\mu_{x y} & \mu_{y y}
\end{array}\right] u\left\|f_{n i}\right\|
$$

where $u$ is the normalized 2D velocity direction, and $\mu_{x x}, \mu_{x y}$, and $\mu_{y y}$ are three friction constants. This model addresses the anisotropic frictional behavior of the object such as high latitudinal friction in a wood beam surface due to longitudinal orientation of its fiber cells [19]. The proposed model is not computationally intensive, and it is obtainable during a pregrasp procedure.
Identification of $\mu_{x x}, \mu_{x y}$, and $\mu_{y y}$ in 2 require at least three different sets of data. Similar to human facing a new object to manipulate, we suggest the gripper inspecting the object friction by touching the surface of the object. The gripper starts inserting a small amount of normal contact force, $\left\|f_{n i}\right\|$, and moves while measuring the reaction force, $\left\|f_{t i}\right\|$. The contact force is considered to be small for damage avoidance and the process is repeated at least in three different directions on the object. Once all three friction constants are available, we have an understanding of the anisotropic friction behavior in different directions.

\section{B. Grasp Quality}

This paper considers the general case for both fully and under actuation of the gripper. A transmission matrix, $T$, is defined to relate actuators torque vector to joints torque vector as follows

$$
t=T^{T} \tau
$$

where $\tau \in R^{n_{q}}$ is the torque vector for a gripper with $n_{q}$ joints, and $t \in R^{n_{a}}$ is the torque vector of $n_{a}$ actuators. The transmission matrix is a unit matrix in a fully actuated gripper. The mapping from contact forces to the robot joint torques for a grasp with $n_{c}$ contact points is $\tau=J^{T} f$ where $f \in R^{3 n_{c}}$ is contact forces vector, and $J \in R^{3 n_{c} \times n_{q}}$ is Jacobian matrix. The defective class of grasping happens when certain contact forces produce no joint torques or vice versa. In other words, there are certain contact force vectors which are inside the left nullspace of the Jacobian matrix $\left(\mathscr{N}\left(J^{T}\right)\right)$.

Any gripper with hard contact and friction can transfer three components of force to the object. Our proposed friction model determines the tangential force component in any direction according to the normal force. Knowing the maximum actuation saturation and having friction model can give the maximum wrench that the robot can exert. Taking both the gripper grasp capability and task-oriented information into account, we propose the following grasp evaluation metric,

$$
Q=\min _{i} \frac{\left\|w_{i, \max }\right\|}{\left\|w_{i, y}\right\|}, i=1, \ldots, n_{t}
$$

where $w_{i, \max }$ is maximal applicable wrench, $w_{i, y}$ is the failing/yielding task vector according to proposition 1 \& $\& 2$ and $n_{t}$ is the number of failing/yielding task vectors. This quality metric entails repeated identification of the maximal wrench that can be applied by the gripper to the object in a given direction defined by a task vector.

Maximizing the applicable wrench in a given direction can be solved as a linear optimization problem. $\left\|w_{i}\right\|$ is the value that we wish to maximize. This maximization problem is subjected to (3), and

$$
d_{i}\left\|w_{i}\right\|=G f
$$

where $d_{i}=\frac{w_{i, y}}{\left\|w_{i, y}\right\|}$. The nullspace of Grasp matrix $(\mathscr{N}(G))$ is the subspace of internal forces. Internal force results in wrench intensity but not object motion. It can be easily shown that internal forces are controllable by joint actions 
TABLE I

Properties of Materials [17], [20]

\begin{tabular}{ll}
\hline \hline Steel Round Beam Radius $(\mathrm{m})$ & 0.003 \\
Steel Young's modulus $\left(\mathrm{N} / \mathrm{m}^{2}\right)$ & $207 \times 10^{9}$ \\
Steel yield strength $\left(\mathrm{N} / \mathrm{m}^{2}\right)$ & $220 \times 10^{6}$ \\
\hline Wood Squire Beam Dimensions $(\mathrm{m} \times \mathrm{m})$ & $0.007 \times 0.007$ \\
Wood Young's modulus $\left(\mathrm{N} / \mathrm{m}^{2}\right)$ & $8.9 \times 10^{9}$ \\
Wood ultimate strength $\left(\mathrm{N} / \mathrm{m}^{2}\right)$ & $40 \times 10^{6}$ \\
\hline \hline
\end{tabular}

if and only if $\mathscr{N}(G) \cap \mathscr{N}\left(J^{T}\right)=0$. (3) and mapped joint torques ensure the quasistatic assumption at the finger joints by equating torques produced by underactuated gripper required to generate the contact forces. (5) ensures that the applied wrench, $G f$, is in the failing/yielding task vector direction. To compute the grasp evaluation metric in (4), we perform the maximization problem. At the end of each maximization step the minimum of $\frac{\left\|w_{i, \max }\right\|}{\left\|w_{i, y}\right\|}$ represents the quality metric.

\section{RESUlTS}

To validate the proposed grasp quality metric, and the friction identification method, failing of both brittle and ductile objects were considered. A circular beam made of steel was considered as an extreme example of ductile material. A wood square beam was considered to demonstrate the validity of the beam profile shape generalization. The properties of these beams is summarized in Table I] The failing location range were given to the grasp planner. The complete failing process was implemented to show the grasp capability.

\section{A. Experimental Setup}

Kuka Light-Weight Robot (LWR) IV and CRS Robotics underactuated gripper were used for conducting the experiments. To exploit the control capabilities of Kuka LWR in parallel use with peripheral tools and sensors, we used the open-source KUKA UI (https://github.com/ mahyaret/KUKA-UI). Load cells in fingers and an ATI 6axis force/torque sensor at the wrist were used for acquiring data (see Fig. 2). The finger structure is separately shown in Fig. 3. The finger tip that was in contact with the object is shown in this figure. The contact region was a plate screwed to the load cell. The load cell was also screwed to the finger fixture which was actuated by the gripper. With such finger structure contact forces were measured by the load cell.

The target object was fixed from one end to the table and the other end kept loose in the air. At each experiment, the gripper closed fingers $5 \mathrm{~mm}$ above the target point. Robot manipulator then oriented the gripper in a planar motion around the target point. In this way, the probable fracture was set to be the $5 \mathrm{~mm}$ region. Robot manipulator moved slowly enough to satisfy the quasistatic assumption.

CRS Robotics gripper is a planar underactuated open/close gripper. The two fingers are actuated dependently by a single

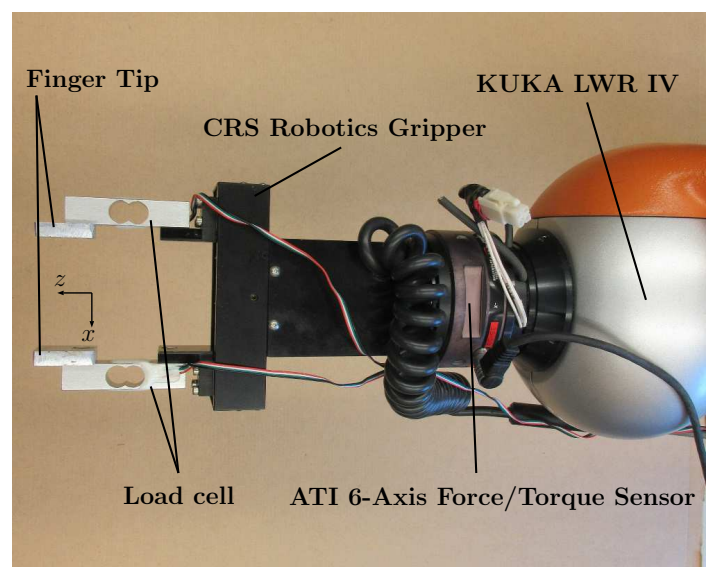

Fig. 2. Experimental hardware setup.

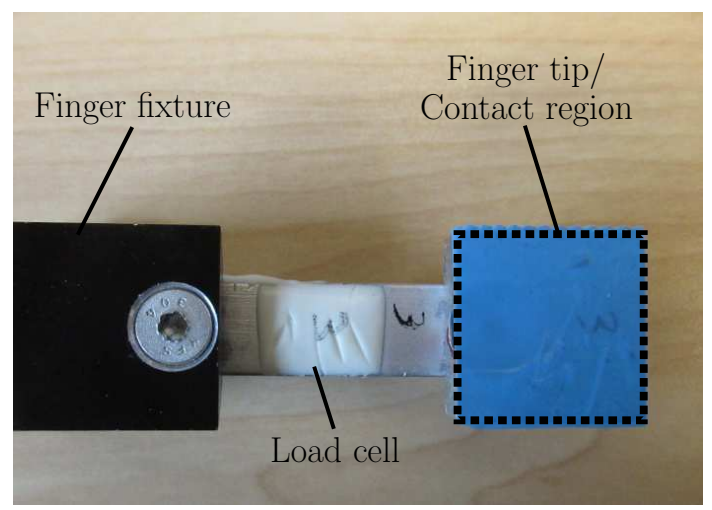

Fig. 3. Finger structure consisting of finger tip plate, load cell, and the fixture. The contact region is the area on the finger tip which is in contact with the object.

DC motor. The Jacobian and transmission matrices for this gripper are,

$$
J=\left[\begin{array}{cccccc}
0 & 0 & 0 & -1 & 0 & 0 \\
1 & 0 & 0 & 0 & 0 & 0
\end{array}\right]^{T}, T=\left[\begin{array}{c}
1 \\
-1
\end{array}\right]
$$

The grasp matrix for this two contact points planar scenario can be easily obtained. Internal forces which are in the nullspace of Grasp matrix, excluding the left nullspace of Jacobian matrix, are simply those that result in squeezing the object, i.e., $A=[-0.7071,0,0,0.7071,0,0]^{T}$.

We used the gripper itself as our frictional test device. The robot fingers applied small magnitude normal forces to the surface of the object while attempting to move along the object in different tangential directions (see Fig. 4). Load cells embedded in the fingers measured the applied normal forces. The tangential force equivalent to friction force was measured using the 6-axis force/torque sensor. In practice, normal forces produce a chattering effect in contact with hard surfaces due to unavoidable measurement noises. A PID controller enhanced with Kalman filter was used to regulate the normal forces. 


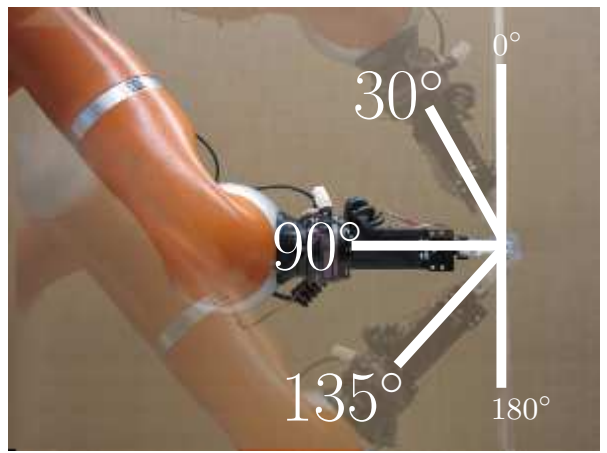

Fig. 4. Tangential directions that the robot fingers apply low magnitude normal forces to the surface of the object while attempting to move upward. Tangential direction of $30^{\circ}, 90^{\circ}$, and $135^{\circ}$.

¿
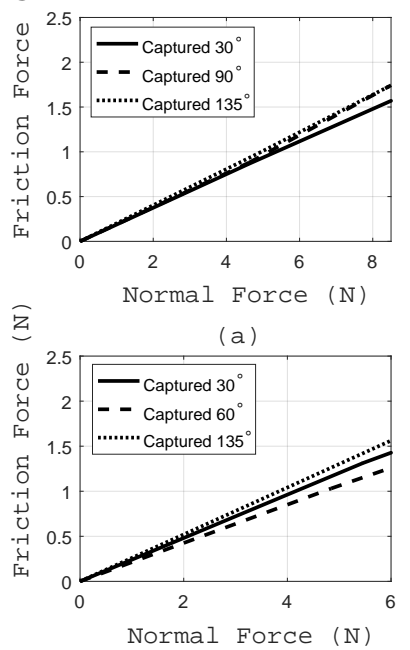

(c)

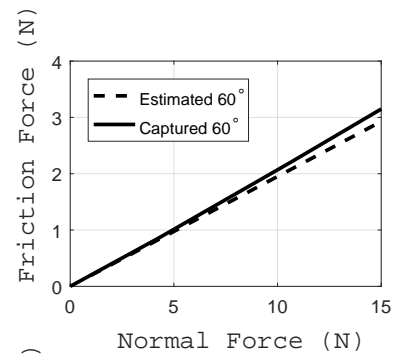

(b)

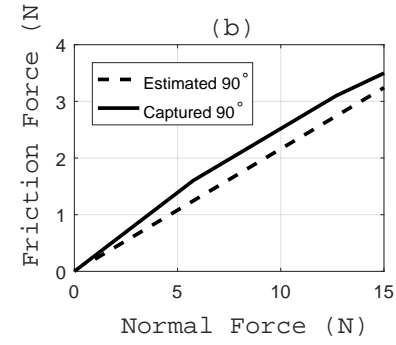

(d)

Fig. 5. Friction test and validation results. (a) Anisotropic behavior of the friction in steel beam. (b) Steel beam friction model validation at $60^{\circ}$. (c) Anisotropic behavior of the friction in wood beam. (d) Wood beam friction model validation at $90^{\circ}$.

\section{B. Experimental Results}

Different normal contact forces were regulated, and the tangential reaction forces were measured. These contact forces were then applied in various orientations. Figure $5 \mathrm{a}, \mathrm{c}$ illustrate the friction forces produced by the finger moving upward exerting different normal forces. These figures compare the friction behavior of the objects when they are grasped with different orientations. These figures depict the anisotropy in friction. It can be seen that steel had more homogeneous behavior than wood as expected. These experiments substantiated the importance of frictional tests.

Using the normal and tangential forces, the friction constants were obtained. Different friction constants for two types of beams are provided in Table 5. Note that $\mu_{x y}$ can be negative while the matrix $\left[\mu_{x x}, \mu_{x y} ; \mu_{x y}, \mu_{y y}\right]$ remains a positive definite matrix. The highest friction direction suggests the orientation for the grasping. The identified friction model was then validated by applying larger normal force and measuring the reaction in new orientations. The

TABLE II

FRICTION CONSTANTS

\begin{tabular}{llll}
\hline \hline Material & $\mu_{x x}$ & $\mu_{x y}$ & $\mu_{y y}$ \\
\hline Steel & 0.2031 & -0.0073 & 0.2106 \\
Wood & 0.2255 & -0.0436 & 0.2639 \\
\hline \hline
\end{tabular}

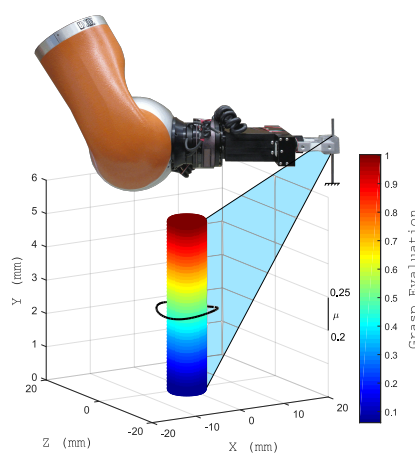

(a)

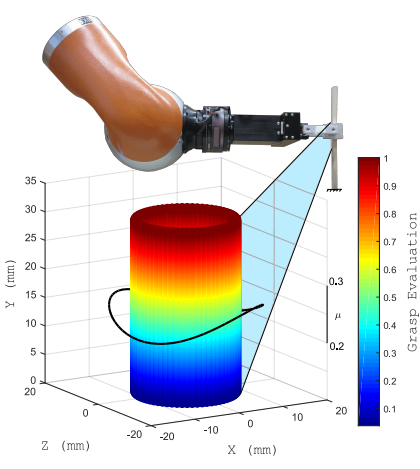

(b)
Fig. 6. Grasp planner results for steel beam, wood beam. The intended yielding location is color coded by the value of grasp evaluation metric. (a) Grasp evaluation for steel beam. The color is slightly changed by the friction change. (b) Grasp evaluation for wood beam. The color is slightly changed by the friction change.

result of this validation is shown in Fig. 5 b,d. It can be seen that we used different angles and even contact forces range for validations to illustrate the capability of the friction model in handling the anisotropic behavior of the object. Note that similar to the human, the friction identification serves as an initial object inspection for the planner; hence, it is meant to be fast without damaging the object surface.

Gripper specifications with measured friction and fracture location with failure task information were fed into the grasp planner to find the best grasp for failing the part. The largest applicable twisting torque is dependent on the highest friction between the gripper and the object, and the normal force insertion capability. Normal force insertion capability is also limiting for applicable bending moment around the intended fracture location. Figure 6 shows grasp planner result. The best locations to grasp are the red area in Fig. 6 which provides the largest leveraged yielding stress. The quality metric for both wood and steel beams were similar since it is mostly dependent on the bending moment arm. Fig. 6 also emphasizes the larger contribution of bending stress in comparison to shear stress.

Figure 6 shows part of the steel beam which was intended for yielding. The moment arm of $5 \mathrm{~mm}$ was considered. The grasp evaluation metric based on the applicable wrench on the steel beam is normalized and color coded in Fig. 6a. Friction variation of the steel beam is illustrated in Fig. 6 a for justification of the slight change in color where friction has the lowest value. The same process was repeated for the virtual cylindrical beam covering the square wood beam with moment arm of $30 \mathrm{~mm}$. As it can be seen from Fig. 6p even larger leverage for torsion in the wood beam did not provide the enough stress for yielding by torsion.

Investigating the high friction direction is necessary for 


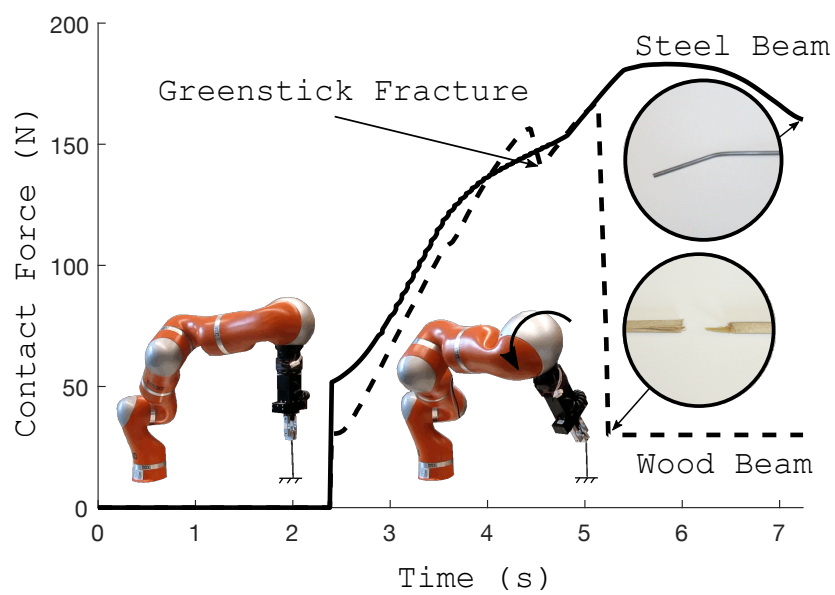

Fig. 7. Failure test results. Yielding steel beam by means of permanently distorting it and Failing wood beam by means of breaking it to pieces. The Greenstick fracture behavior can be explained by anisotropy between the radial and tangential directions.

applying tangential forces needed in twisting and/or bending. Note that the suggested identification procedure is meant to be fast and provide the high friction direction for the grasp planner. As it was shown in Fig. 6p, wood surface has noticeable high friction in certain directions which can be explained by anisotropy between the radial and tangential directions.

To validate the suggested grasp area by the grasp planner, we implemented the complete failure of the objects. Figure 7 depicts the measured reaction contact force while failing the objects. By continuously orienting the gripper around the predetermined yielding location, a larger reaction contact force was sensed as shown in Fig. 7 for steel beam until the distortion became permanent and the resisting moment dropped. The normal stress in this process for steel beam was $2.4770 \times 10^{8} \mathrm{~N} / \mathrm{m}^{2}$ which is larger than its yielding strength. In a wood beam, snapping occurred after the Greenstick fracture effect was observed as shown in Fig. 7 the contact forces dropped once before dropping to grasping force. The normal stress for wood beam was calculated as $4.3163 \times 10^{7} \mathrm{~N} / \mathrm{m}^{2}$ which is more than its ultimate strength.

\section{CONCLUSiOnS AND Future Works}

This paper considered the problem of purposefully failing/yielding an object in robotic harvesting. It was shown via mechanical failure theories and experimental results that leverage based bending produced more effective failure stress. Additionally, it was also theoretically discussed that if a large twisting arm was available, torsion could also be effective. This is the exact case of robotic harvesting, where fruits provide a long twist arm around the stem. We examined the part before grasping for measuring the friction between the gripper and the object (for better understanding watch: https://youtu.be/4XH8ZRJO_b8). The friction modeling and measurement experiment allowed us to obtain the capability of the gripper in twisting torque insertion. The model included more complex behavior such as anisotropy. The future work will be considering the effect of natural material compliance on the stiffness and stability of frictional multi-contact grasps and fixtures.

\section{REFERENCES}

[1] A. Van Casteren, W. Sellers, S. Thorpe, S. Coward, R. Crompton, and A. Ennos, "Why don't branches snap? the mechanics of bending failure in three temperate angiosperm trees," Trees, vol. 26, no. 3, pp. 789-797, 2012

[2] A. Bicchi and V. Kumar, "Robotic grasping and contact: A review," in Robotics and Automation, 2000. Proceedings. ICRA'O0. IEEE International Conference on, vol. 1. IEEE, 2000, pp. 348-353.

[3] A. Sahbani, S. El-Khoury, and P. Bidaud, "An overview of 3d object grasp synthesis algorithms," Robotics and Autonomous Systems, vol. 60, no. 3, pp. 326-336, 2012.

[4] J. Bohg, A. Morales, T. Asfour, and D. Kragic, "Data-driven grasp synthesisa survey," IEEE Transactions on Robotics, vol. 30, no. 2, pp. 289-309, 2014

[5] F. Veiga, H. van Hoof, J. Peters, and T. Hermans, "Stabilizing novel objects by learning to predict tactile slip," in Intelligent Robots and Systems (IROS), 2015 IEEE/RSJ International Conference on, Sept 2015, pp. 5065-5072.

[6] X. A. Wu, N. Burkhard, B. Heyneman, R. Valen, and M. Cutkosky, "Contact event detection for robotic oil drilling," in Robotics and Automation (ICRA), 2014 IEEE International Conference on, May 2014, pp. 2255-2261.

[7] G. D. Maria, P. Falco, C. Natale, and S. Pirozzi, "Integrated force/tactile sensing: The enabling technology for slipping detection and avoidance," in Robotics and Automation (ICRA), 2015 IEEE International Conference on, May 2015, pp. 3883-3889.

[8] M. AbdeEtedal, H. Talebi, and F. Abdollahi, "Scale-dependent method for whole arm grasp evaluation," in IECON 2012-38th Annual Conference on IEEE Industrial Electronics Society. IEEE, 2012, pp. 2780-2785.

[9] C. Borst, M. Fischer, and G. Hirzinger, "Grasp planning: How to choose a suitable task wrench space," in Robotics and Automation 2004. Proceedings. ICRA'04. 2004 IEEE International Conference on, vol. 1. IEEE, 2004, pp. 319-325.

[10] Z. Li and S. S. Sastry, "Task-oriented optimal grasping by multifingered robot hands," IEEE Journal on Robotics and Automation, vol. 4, no. 1 , pp. 32-44, 1988

[11] X. Zhu and J. Wang, "Synthesis of force-closure grasps on 3-d objects based on the q distance," IEEE Transactions on robotics and Automation, vol. 19, no. 4, pp. 669-679, 2003.

[12] L. Han, J. C. Trinkle, and Z. X. Li, "Grasp analysis as linear matrix inequality problems," IEEE Transactions on Robotics and Automation, vol. 16, no. 6, pp. 663-674, 2000.

[13] T. Callier, H. P. Saal, E. C. Davis-Berg, and S. J. Bensmaia, "Kinematics of unconstrained tactile texture exploration," Journal of neurophysiology, vol. 113, no. 7, pp. 3013-3020, 2015.

[14] T. Yoshioka, J. C. Craig, G. C. Beck, and S. S. Hsiao, "Perceptual constancy of texture roughness in the tactile system," The Journal of Neuroscience, vol. 31, no. 48, pp. 17603-17611, 2011.

[15] M. Janko, R. Primerano, and Y. Visell, "On frictional forces between the finger and a textured surface during active touch," IEEE transactions on haptics, 2015.

[16] A. Tekkaya, J. Allwood, P. Bariani, S. Bruschi, J. Cao, S. Gramlich, P. Groche, G. Hirt, T. Ishikawa, C. Löbbe et al., "Metal forming beyond shaping: Predicting and setting product properties," CIRP Annals-Manufacturing Technology, vol. 64, no. 2, pp. 629-653, 2015.

[17] J. E. Shigley, Shigley's mechanical engineering design. Tata McGrawHill Education, 2011.

[18] M. Abdeetedal and M. R. Kermani, "Optimal grasp synthesis to apply normal and shear stresses of failure in beams," in Advanced Intelligent Mechatronics (AIM), 2016 IEEE International Conference on. IEEE, 2016, pp. 395-400.

[19] A. Reiterer, I. Burgert, G. Sinn, and S. Tschegg, "The radial reinforcement of the wood structure and its implication on mechanical and fracture mechanical properties - a comparison between two tree species," Journal of Materials Science, vol. 37, no. 5, pp. 935-940, 2002.

[20] R. M. Rowell, Handbook of wood chemistry and wood composites. CRC press, 2012 